\title{
BIMODAL MYOCARDIAL MOTION ANALYSIS FROM B-MODE AND TISSUE DOPPLER ULTRASOUND
}

\author{
Michael Sühling, Muthuvel Arigovindan, Christian Jansen*, Patrick Hunziker*, and Michael Unser \\ Swiss Federal Institute of Technology Lausanne \\ Biomedical Imaging Group, STI/BIO-E \\ *University Hospital Basel \\ CH-1015 Lausanne, Switzerland \\ Medical Intensive Care Unit \\ CH-4031 Basel, Switzerland
}

\begin{abstract}
We present a new method for estimating heart motion from two-dimensional echocardiographic sequences by exploiting two ultrasound modalities: B-mode and tissue Doppler. The algorithm estimates a two-dimensional velocity field locally by using a spatial affine velocity model inside a sliding window. Within each window, we minimize a local cost function that is composed of two quadratic terms: an optical flow constraint that involves the B-mode data and a constraint that enforces the agreement of the velocity field with the directional tissue Doppler measurements. The relative influence of the two different modalities to the resulting solution is controlled by an adjustable weighting parameter. Robustness is achieved by a coarse-to-fine multi-scale approach.

The method was tested on synthetic ultrasound data and validated by a rotating phantom experiment. First applications to clinical echocardiograms give promising results.
\end{abstract}

\section{INTRODUCTION}

Echocardiography is an established clinical tool for the assessment of regional ventricular function in patients with cardiac disease. B-mode and tissue Doppler modalities, in particular, are widely used to analyze myocardial motion in order to evaluate the degree of ischemia and infarction. B-mode echocardiograms provide a two-dimensional gray-scale display of the moving heart but no quantitative motion information. Therefore, the assessment of myocardial motion form B-mode echocardiograms is routinely accomplished by visual interpretation and manual evaluation. This leads to subjective and, at best, semi-quantitative diagnoses, which suffer from a significant intra- and interobserver variability.

Tissue Doppler imaging (TDI) is an emerging ultrasound modality that has been introduced recently as a mod-

E-mail: michael.suehling@epfl.ch, muthuvel.arigovindan@epfl.ch, christian.jansen@unibas.ch, PHunziker@uhbs.ch, michael.unser@epfl.ch. This work was supported in part by the Swiss National Science Foundation under grant 3200-059517.99 and the Swiss Heart Foundation. ification of Doppler-based blood flow measurement [1]. It analyzes high-amplitude, low-frequency Doppler shifts of reflected echoes to quantify tissue motion. Among others, TDI has been used to detect wall motion abnormalities in stress echocardiography [2]. Low Doppler velocities have proven to be a strong indicator for ischemia [3]. However, TDI measures only the velocity component along the ultrasonic beam direction. The fact that ventricular motion is multi-dimensional and usually not restricted to the scan line makes its interpretation difficult-especially since the images are scanned in a radial fashion.

To obtain true two-dimensional, quantitative motion information, different methods have been proposed to estimate ventricular motion from B-mode echocardiograms [4]. In this paper, we propose a new motion analysis approach that is based on both modalities, B-mode and tissue Doppler. The idea is to estimate a velocity field from Bmode echocardiograms such that its projections along the scan lines are in good agreement with the tissue Doppler measurements.

The paper is organized as follows. In Section 2, we briefly review the principles of tissue Doppler imaging. After describing the optical flow principle to analyze motion from B-mode echocardiograms in Section 3, we present the proposed bimodal algorithm in Section 4. Numerical results from synthetic data, phantom experiments and clinical echocardiograms are given in Section 5.

\section{PRINCIPLES OF TISSUE DOPPLER IMAGING}

Velocity measurements using pulsed-wave ultrasound have become an important diagnostic tool in echocardiography $[3,2]$. In two-dimensional tissue Doppler imaging (TDI), the beam is scanned radially over the region to be imaged. As illustrated in Fig. 1, the system measures the tissue velocity component

$$
v_{D}=\|\mathbf{v}\|_{2} \cos (\beta)
$$

at positions along each beam, where $\beta$ denotes the angle between the true velocity vector $\mathbf{v}$ and the beam direction $\alpha$. 


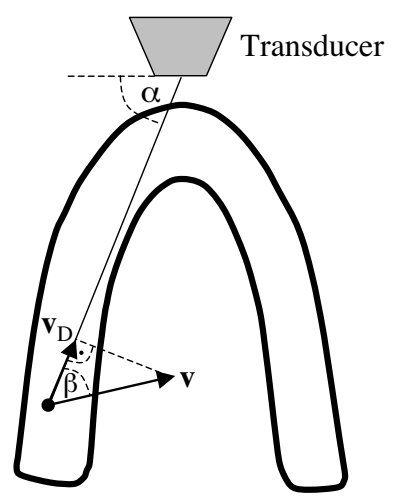

Fig. 1. The tissue Doppler velocity $\mathbf{v}_{D}$ corresponds to the projection of the true velocity $\mathbf{v}$ along the beam direction $\alpha$.

Thus, the measured motion depends highly on $\beta$. In particular, the modality is totally blind to displacements orthogonal to the scan line.

Conventional Doppler ultrasonography is based on the principle that $v_{D}$ is proportional to the small frequency shift $f_{D}$ in the ultrasound carrier frequency $f_{0}$ between transmitted and received echoes, i.e.,

$$
v_{D}=\frac{c}{2 f_{0}} f_{D}
$$

where $c$ denotes the speed of sound.

Current ultrasound systems allow the simultaneous acquisition of B-mode and tissue Doppler signals in realtime. The Doppler velocities are usually color-coded and superimposed onto the B-mode echocardiogram as shown in Fig. 5(b). However, the interpretation of these radial projections of complex motion patterns, such as translation, rotation and shear, is difficult and requires a sufficient level of experience [2].

\section{MOTION ANALYSIS FROM B-MODE ECHOCARDIOGRAMS}

Since tissue Doppler imaging is limited to the ultrasonic beam direction, several methods have been proposed to estimate true two-dimensional motion from dynamic B-mode echocardiograms; optical flow methods, in particular, have led to promising results [4]. Gradient-based optical flow estimation relies on the assumption that the intensity of a particular point in a moving pattern does not change with time. Let $I(x, y, t)$ denote the intensity of pixels at location $\mathbf{r}=(x, y)$ and time $t$ in an image sequence. Then the constant intensity assumption can be expressed as [5]

$$
I_{x}(\mathbf{r}, t) u(\mathbf{r}, t)+I_{y}(\mathbf{r}, t) v(\mathbf{r}, t)+I_{t}(\mathbf{r}, t)=0,
$$

where $I_{x}, I_{y}$ and $I_{t}$ denote the spatial and temporal derivatives of the image intensity. To obtain accurate numerical derivative estimates in our experiments, we use cubic spline interpolation [6] in space and time. The velocities $u$ and $v$ are, respectively, the $x$ - and $y$-components of the optical flow that we wish to estimate. Since (1) is a single equation in two unknowns $u$ and $v$, it cannot be solved uniquely without introducing additional constraints. The Lucas-Kanade method [4], for instance, assumes the velocity to be constant within small spatial neighborhoods. It has been applied to echocardiograms by Chunke et al. [7].

\section{BIMODAL MOTION ANALYSIS FROM B-MODE AND TISSUE DOPPLER ECHOCARDIOGRAMS}

Since both modalities, B-mode and tissue Doppler, provide valuable motion information, we propose a novel algorithm that integrates these two kinds of information to estimate a true two-dimensional velocity field. Inspired by the LucasKanade method, we propose a sliding-window algorithm. Since typical heart motions are given by rotation, expansion, contraction, and shear, we use a local affine model for the velocity in space. Let $\mathbf{r}_{0}=\left(x_{0}, y_{0}\right)^{T}$ denote the center of a small image region for a frame at time $t$. Omitting the temporal parameter for notational convenience, the spatial affine model is defined as

$$
\mathbf{v}(\mathbf{r})=\mathbf{v}_{0}+\left(\begin{array}{ll}
u_{x} & u_{y} \\
v_{x} & v_{y}
\end{array}\right) \cdot\left(\mathbf{r}-\mathbf{r}_{0}\right),
$$

where $\mathbf{r}=(x, y)^{T}$ and $\mathbf{v}(\mathbf{r})=(u(\mathbf{r}), v(\mathbf{r}))^{T}$. The vector $\mathbf{v}_{0}$ corresponds to the velocity at the center point $\mathbf{r}_{0} ; u_{x}, u_{y}$, $v_{x}$, and $v_{y}$ are the first order spatial derivatives of $u$ and $v$, respectively. These derivatives are assumed to be constant within the local neighborhood.

We estimate the local model parameters by minimizing the weighted least-squares criterion

$$
\begin{aligned}
& \sum_{n} w\left(\mathbf{r}_{n}-\mathbf{r}_{0}\right)\left(\left(I_{x}\left(\mathbf{r}_{n}\right) u\left(\mathbf{r}_{n}\right)+I_{y}\left(\mathbf{r}_{n}\right) v\left(\mathbf{r}_{n}\right)+I_{t}\left(\mathbf{r}_{n}\right)\right)^{2}\right. \\
& \left.+\lambda\left(\cos \left(\alpha_{n}\right) u\left(\mathbf{r}_{n}\right)+\sin \left(\alpha_{n}\right) v\left(\mathbf{r}_{n}\right)-v_{D}\left(\mathbf{r}_{n}\right)\right)^{2}\right)
\end{aligned}
$$

where the sum is taken over all pixels $\mathbf{r}_{n}$ inside an observation window $w$ centered at position $\mathbf{r}_{0}$. The first term of the cost function is equal to the optical flow constraint applied to the B-mode data. The second term corresponds to the difference between the measured tissue Doppler velocities and the velocity field projection along the scan line. The scan line direction at position $\mathbf{r}_{n}$ is given by the unit vector $\left(\cos \left(\alpha_{n}\right), \sin \left(\alpha_{n}\right)\right)$. The relative influence of the Bmode data versus the tissue Doppler measurements to the resulting velocity estimate is controlled by the non-negative weighting parameter $\lambda$. The symmetric window function $w$ gives more weight to constraints at the center of the local spatial region than to those at the periphery. A well suited window function is $w(x, y)=\beta^{n}(x) \beta^{n}(y)$, where $\beta^{n}$ is 


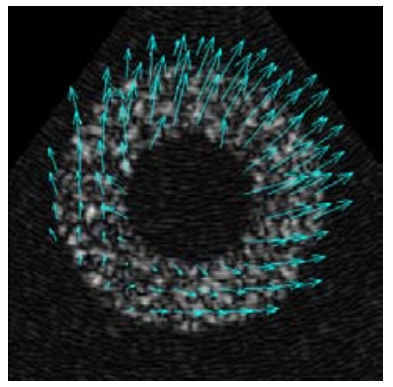

(a) B-mode echocardiogram with superimposed estimated velocity field

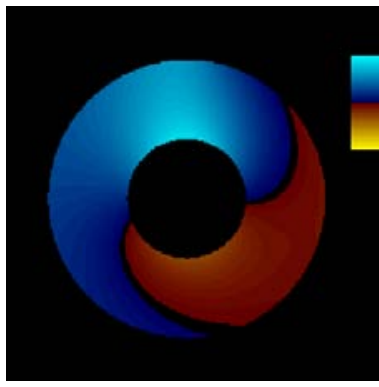

(b) Color-coded simulated tissue Doppler velocities
Fig. 2. Frame of synthetic echocardiogram during diastole with superimposed velocity information.

the symmetrical B-spline of degree $n \in \mathbb{N}$ [6]. B-splines rapidly converge to Gaussians when their degree increases which ensures isotropy of the window in multiple dimensions.

By inserting (2) into (3) and differentiating the latter one with respect to each of the six unknown model parameters $\mathbf{x}=\left(u_{0}, v_{0}, u_{x}, u_{y}, v_{x}, v_{y}\right)^{T}$, we obtain a symmetric linear system $\mathbf{A x}=\mathbf{b}$ of size $(6 \times 6)$ that has to be solved at each window position.

\subsection{Coarse-To-Fine Multi-Scale Strategy}

It is obviously difficult to estimate large motions at fine scales. A way around this problem is to apply a coarseto-fine strategy. At each spatial scale $j$, we use dilated and shifted versions of the window function:

$$
w^{(j)}\left(x-x_{0}, y-y_{0}\right)=w\left(\frac{x-2^{j} x_{0}}{2^{j}}, \frac{y-2^{j} y_{0}}{2^{j}}\right) .
$$

The window functions at scale $j$ are dilated by the factor $2^{j}$ and are spaced at a distance of $2^{j}$ pixels.

The motion vectors are propagated to the next finer resolution level as initial estimates and are then replaced if they do not already exceed a scale-dependent size. For each local estimate, we compute a confidence measure. A local estimate is replaced only if its confidence measure is larger than the corresponding one at the next coarser scale. Otherwise, the coarser scale estimator is kept. Furthermore, a solution of a local linear system is regarded as not admissible if the linear system is either ill-conditioned or if the length of the estimated central motion vector exceeds some scale-dependent limit. A motion estimate is set to zero if the local mean of the time derivative at the given location is below a pre-defined noise level. Finally, the discrete motion

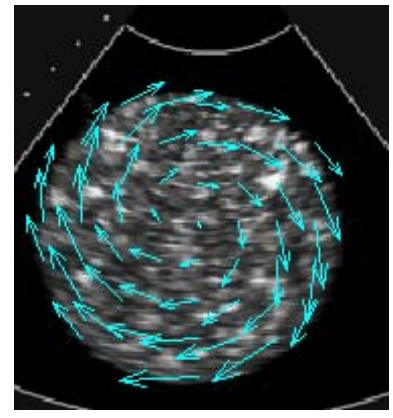

(a) B-mode echocardiogram with superimposed estimated velocity field

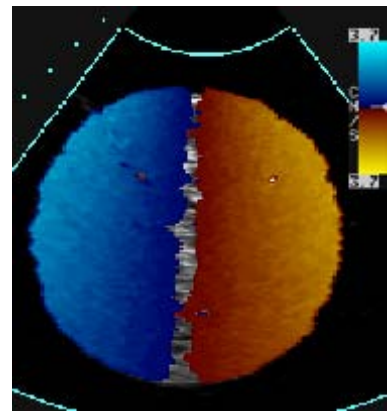

(b) B-mode echocardiogram with superimposed colorcoded Doppler velocities
Fig. 3. Frame of rotating phantom echocardiogram with superimposed velocity information.

estimates at the finest scale $j_{0}$ are interpolated by B-splines to obtain a continuous representation of the motion field.

\section{NUMERICAL RESULTS}

For validation purposes, the algorithm was tested on synthetic data, on ultrasound sequences of a rotating phantom, and on clinical echocardiograms. All echocardiograms were acquired with a HP Sonos 5500 ultrasound system.

\subsection{Application to Synthetic Data}

The algorithm was first tested on synthetic ultrasound sequences for which the exact motion field and corresponding tissue Doppler velocities are known. The data was generated by warping a single reference frame of point scatterers. To simulate noise, we added scatterers of random echogenicity to each frame. The final B-mode images were obtained by applying a simple, linear ultrasound imaging model to the perturbed point scatterer images. The example sequence used here simulates a full cardiac cycle of a left ventricular short axis view (SAX) with an underlying translation to the upper right. Fig. 2(a) shows one diastolic frame of the sequence together with the superimposed estimated velocity field. The color-coded simulated tissue Doppler velocities are shown in Fig. 2(b).

To assess the performance of the algorithm, we use an angular error measure between the estimated velocity $\widehat{\mathbf{v}}$ and the exact velocity $\mathbf{v}$ which is given by $\theta=\arccos (<\mathbf{v}, \widehat{\mathbf{v}}\rangle$ $\left./\left(\|\mathbf{v}\|_{2}\|\widehat{\mathbf{v}}\|_{2}\right)\right)$. The mean angular error $\bar{\theta}$ is computed by averaging $\theta$ over the whole image sequence. Fig. 4(a) illustrates the average error for different values of the parameter $\lambda$ that controls the relative influence of B-mode versus tissue Doppler data in (3). First, the mean error decreases 


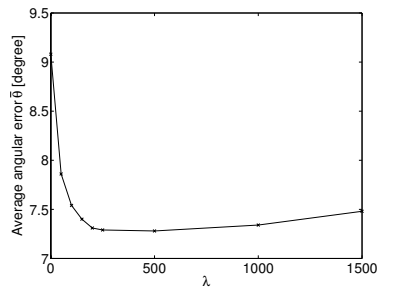

(a) Angular error for synthetic ultrasound data

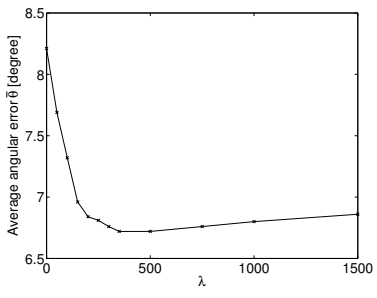

(b) Angular error for phantom sequence
Fig. 4. Average angular error $\bar{\theta}$ for different values of $\lambda$ computed for synthetic and phantom ultrasound data.

significantly with increasing weight of the tissue Doppler term. The relative error improvement between $\lambda=0$ (only B-mode data is used) and $\lambda=500$ is $19.82 \%$. For larger values of $\lambda$, the error increases again and the local linear systems become more and more ill-conditioned.

\subsection{Phantom Experiments}

The algorithm's ability to analyze motion from real ultrasound data was tested by a phantom experiment. A cylindershaped, tissue-mimicking phantom was placed inside a tube of water and rotated with constant angular velocity around its longitudinal axis. Fig. 3(a) shows one frame of the Bmode sequence together with the superimposed estimated velocity field. Fig. 3(b) shows the measured tissue Doppler velocities. The central vertical line of zero Doppler velocities clearly demonstrates that motion orthogonal to the beam direction cannot be measured by TDI, whereas the proposed algorithm is independent from the beam direction. The average angular error $\bar{\theta}$ in dependency of $\lambda$ is plotted in Fig. 4(b). The optimal error improvement of $18.15 \%$ is obtained for $\lambda=350$. As in the case of synthetic data, the error increases if either of the two modalities becomes dominant.

\subsection{Application to Clinical Data}

For a first in vivo validation, we applied the method to a set of clinical echocardiograms. Fig. 5(a) shows one frame of a B-mode sequence during systole. The corresponding estimated velocity field is superimposed. In contrast to TDI, that measures mainly the contraction towards the apex (Fig. 5(b)), the estimated motion field also captures the significant inward motion of the cardiac walls. This corresponds well to the expert echocardiographic reading.

\section{CONCLUSION}

We have proposed a new method to estimate heart motion from echocardiograms that combines information from B-

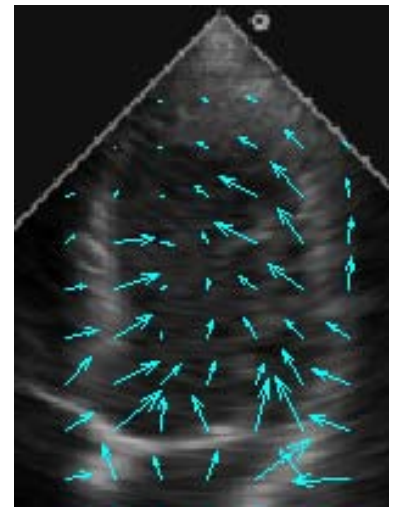

(a) B-mode echocardiogram estimated velocity field with superimposed

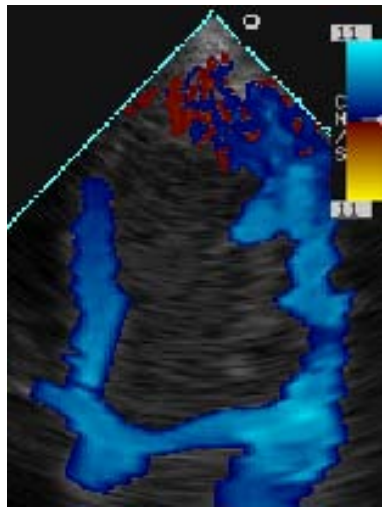

(b) B-mode echocardiogram with superimposed colorcoded Doppler velocities
Fig. 5. Frame of clinical echocardiogram with superimposed velocity information during systole.

mode and tissue Doppler modalities. Validation experiments on synthetic and phantom data demonstrate that the inclusion of tissue Doppler measurements significantly improves the motion field accuracy. First applications to clinical echocardiograms also give realistic results.

\section{REFERENCES}

[1] G. Sutherland, M. Steward, K. Grounstroem, et al., "Color doppler myocardial imaging: a new technique for assessment of myocardial function," J. Am. Soc. Echocardiogr., vol. 7, no. 5, pp. 441-458, Sept.-Oct. 1994.

[2] P. Hunziker, M. Picard, N. Jander, et al., "Regional wall motion assessment in stress echocardiography by tissue Doppler bull's-eyes." J. Am. Soc. Echocardiography, vol. 12, no. 3, pp. 196-202, Mar. 1999.

[3] G. Derumeaux, M. Ovize, J. Loufoua, et al., "Doppler tissue imaging quantitates regional wall motion during myocardial ischemia and reperfusion," Circulation, vol. 97, no. 19, pp. 1970-1977, May 1998.

[4] P. Baraldi, A. Sarti, C. Lamberti, et al., "Evaluation of differential optical flow techniques on synthesized echo images," IEEE Trans. Biomed. Eng., vol. 43, no. 3, pp. 259-272, Mar. 1996.

[5] B. Horn and B. Schunk, "Determining optical flow," Artificial Intelligence, vol. 17, pp. 185-203, 1981.

[6] M. Unser, "Splines: A perfect fit for signal and image processing," IEEE Signal Processing Mag., vol. 16, no. 6, pp. 22-38, Nov. 1999.

[7] Y. Chunke, K. Terada, and S. Oe, "Motion analysis of echocardiograph using optical flow method," IEEE International Conference on Systems, Man and Cybernetics, vol. 1, pp. 672677, 1996. 